\title{
Refractory Status Epilepticus in Children: When Do We Stop Treatment?
}

\author{
N.K. Sethi ${ }^{\mathrm{a}} \quad$ J. Torgovnick $^{\mathrm{b}} \quad$ E. Arsura ${ }^{\mathrm{c}}$ \\ ${ }^{a}$ Comprehensive Epilepsy Center, NYP-Weill Cornell Medical Center, ${ }^{b}$ Saint Vincent's Hospital and Medical Centers, \\ New York, N.Y., and 'Saint Vincent's Hospital and Medical Centers, Staten Island, N.Y., USA
}

\section{Dear Sir,}

Refractory status epilepticus refers to sustained seizures that do not respond to intensive pharmacological interventions with multiple agents. Seizures continue unabated and refractory status epilepticus is associated with elevated morbidity and mortality. Survivors may remain with significant and at times devastating neurological sequelae. Decisions regarding when to terminate care are complex, especially in the pediatric age group, where it is difficult to prognosticate the outcome for a meaningful neurological recovery. A previously healthy 3 -year-old boy presented to our hospital with a new onset seizure occurring in the setting of a febrile illness. While in the emergency room, he had 2 additional generalized tonic-clonic convulsions necessitating intravenous benzodiazepine and phenytoin loading and transfer to the pediatric intensive care unit. While in the pediatric intensive care unit his seizures continued. A loading dose of phenobarbital was given and endotracheal intubation for airway protection was performed. Continuous video-EEG monitoring was started which revealed ongoing generalized electrographic seizures. Intravenous midazolam and pentobarbital led to suppression of all electrographic activity and he remained in this pharmacological-induced suppression burst pattern for 7 days. Electrographic and clinical seizures recurred once sedation was weaned. Over the following months, he continued to manifest subtle clinical signs of going seizures breaking through the intravenous midazolam and pentobarbital suppression. These consisted of clonic eye and limb jerks which were time-locked to the spikes noted during the burst suppression pattern.

Comprehensive workup for etiology of status epilepticus proved noncontributory. Initial magnetic resonance imaging of the brain (MRI) and cerebrospinal fluid sampling revealed no evidence of an infectious or inflammatory etiology. Metabolic screen came back negative for any inborn error of metabolism. There was no evidence to suggest an organic aciduria, glycogen, lysosomal, peroxisomal or urea cycle disorder. As multiple anticonvulsants (including phenytoin, phenobarbital, topiramate, levetriacetam and lamotrigine) failed to cause the seizures to remit, empirical treatment was attempted with pyridoxine, intravenous immunoglobin as well as corticosteroids.

When we first attempted writing this case report, 3.5 months after his first presentation to our emergency room, he continued in a refractory status epilepticus. Multiple attempts to abort the status by induction with pentobarbital, midazolam, propofol and ketamine had failed as well as the use of inhalation anesthetic (isoflurane). After undergoing a subtotal corpus callosotomy, his seizure character changed. His seizures were now more subtle and consisted of intermittent clonic right eyelid jerks along with jerks of the corner of the mouth. Our young patient's intensive care unit stay was complicated with sepsis, a large suboccipital decubitus ulcer and subclavian vein thrombosis. Prolonged ketogenic diet led to significant loss of weight and muscle bulk. VideoEEG now suggested a multifocal origin of his seizures, independently from the right and left hemisphere. Repeat MRI showed extensive cortical atrophy as well as hypoxic and ischemic damage to the deep gray nuclei (fig. 1).

This brings us to the question of when to terminate treatment. Does this child, who has been seizing for more than 3

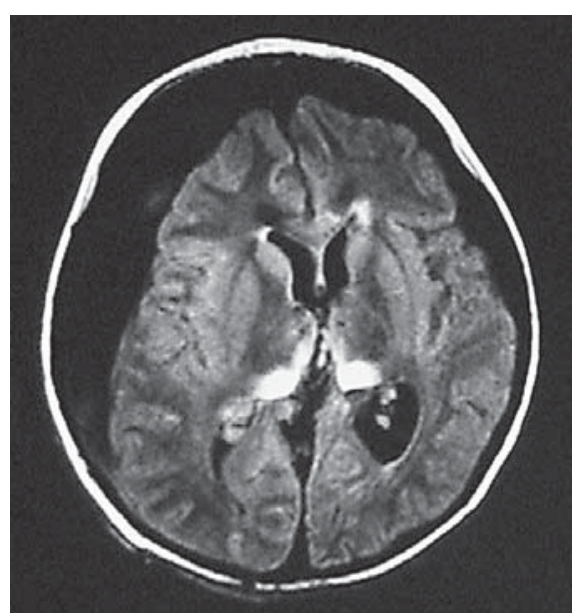

Fig. 1. Axial $\mathrm{T}_{1}$-weighted FLAIR MRI brain showing significant cerebral atrophy and bilateral subdural hygromas.

\section{KARGER}

Fax +41613061234 E-Mail karger@karger.ch www.karger.com
(C) 2007 S. Karger AG, Basel 0014-3022/08/0592-0094\$24.50/0

Accessible online at: www.karger.com/ene
Nitin Sethi, MD

Clinical Neurophysiology Fellow, NYP-Weill Cornell Medical Center 525 East, 68th Street

New York, NY 10021 (USA)

Tel. +1 646515 5168, E-Mail sethinitinmd@hotmail.com 
months, have any chance of a meaningful neurological recovery? Are the parents' expectations reasonable? Meaningful neurological recovery may have different implications for the parents and the treating healthcare professionals. To the parents his very survival may imply meaningful neurological recovery, while to his physicians it is based on more objective assessments of being independent in activities of daily living and free from cognitive deficits. Unfortunately there are no guidelines regarding when to terminate care in pediatric refractory status epilepticus patients. Deciding to terminate 'active' treatment for seizures and instituting palliative care is difficult for all involved. When the decision to terminate active treatment is made the assumption is that further treatment shall not benefit the patient. As death is the expected outcome from terminating treatment, in a way the intention is the death of the patient [1]. Herein is the dilemma pitting this medical collusion with death against the ethical, legal and societal values both of the doctor and the parents. The parents especially may be haunted for life by the guilt that they were responsible in some way for the death of their child. Would we as doctors be facing the same moral dilemma if our patient was an 85year man with multiple medical problems? Does the fact that our patient is a 3-yearold child warrant our medical and ethical obligations to change accordingly? Is there any consensus about terminating treatment when it comes to children? A competent adult can make decisions about endof-life care; about withholding treatment, intubation and tube feeding while in chil- dren usually the parents are obligated and accorded the authority to make these decisions in keeping with their cultural values and moral judgments about what is best for their child [2]. The death of a child evokes deep emotions of grief and anger in the parents and can easily pit them against doctors and nurses.

So where do we go in our case? A frank discussion with the parents regarding the futility of continued treatment for seizures shall be the first step. Explaining to them that terminating active treatment for seizures does not equate to terminating care for their child is paramount and emphasizing that the best nursing and medical care shall continue is paramount. The next 'active' intervention would be to terminate video-EEG monitoring. As epileptologists for the last 3 months we have been using terms like seizures are stable, less frequent or more frequent, all which have gone a long way in confusing the parents and keeping their hopes of a miracle alive. Moving the child out of the pediatric intensive care unit to a quiet single room, giving the parents increased access to touching and interacting with their child, continuing the antiepileptics and aiming for supratherapeutic levels of phenobarbital $(>100 \mu \mathrm{g} / \mathrm{ml})$ while keeping the child sedated and initiating a morphine drip may then be attempted.

Unfortunately there are no guidelines for physicians faced with these dilemmas. Our patient died 4.5 months after his first presentation to our emergency room. Immediate cause of death was attributed to heart failure due to overwhelming sepsis. Could we have done anything differently to provide him with a more dignified death and avoid the tremendous suffering which he and his parents endured? Should we have stopped treatment earlier? Maybe Kenny Rogers rightly summed it for us in his song 'The Gambler':

You got to know when to hold em, know when to fold em,

Know when to walk away and know when to run ...

Now evry gambler knows that the secret to survivin

Is knowin what to throw away and knowing what to keep.

Cause evry hands a winner and evry hands a loser,

And the best that you can hope for is to die in your sleep.

Physicians dealing with older patients often prognosticate as to whether a patient shall leave the hospital alive. Discussions can then be held with the family about termination of care. Children should be treated similarly. Children like adults have the right to a dignified death free of suffering. The above article is our attempt at understanding and making sense of these difficult moral questions, which arose during our care of this sick child.

\section{References}

1 Sayers GM, Perera S: Withholding life prolonging treatment and self-deception. J Med Ethics 2002;28:347-352.

2 Burns JP, Mitchell C: Is there any consensus about end of life care in pediatrics? Arch Pediatr Adolesc Med 2005;159:889-891. 